\title{
Micellar Antibiotics of Bacillus
}

William T. Ferreira ${ }^{1}{ }^{\oplus}$, Huynh A. Hong ${ }^{1}$, Mateusz Hess ${ }^{1}$, James R. G. Adams ${ }^{1}$, Hannah Wood ${ }^{2}$, Karolina Bakun ${ }^{2}$, Sisareuth Tan ${ }^{3}$, Loredana Baccigalupi ${ }^{4}$, Enrico Ferrari ${ }^{5}{ }^{\circledR}$, Alain Brisson ${ }^{3}$, Ezio Ricca ${ }^{6}$, María Teresa Rejas ${ }^{7}$, Wilfried J. J. Meijer ${ }^{7}$, Mikhail Soloviev ${ }^{1}(1)$ and Simon M. Cutting ${ }^{1,2, *}$

1 Department of Biological Sciences, Royal Holloway University of London, Egham TW20 0EX, UK; wuaz082@live.rhul.ac.uk (W.T.F.); hong.huynh@rhul.ac.uk (H.A.H.); mateusz.hess@kcl.ac.uk (M.H.); ja01329@surrey.ac.uk (J.R.G.A.); mikhail.soloviev@rhul.ac.uk (M.S.)

2 SporeGen Ltd., London Bioscience Innovation Centre, 2 Royal College Street, London NW1 0NH, UK; h.wood@sporegen.com (H.W.); k.bakun@sporegen.com (K.B.)

3 Laboratoire d'Imagerie Moléculaire et Nano-Bio-Technologie, UMR-CBMN CNRS-Université de Bordeaux-IPB, 33607 Pessac, France; s.tan@u-bordeaux.fr (S.T.); alain.brisson@u-bordeaux.fr (A.B.)

4 Department of Molecular Medicine and Medical Biotechnology, Federico II University of Naples, 80126 Napoli, Italy; loredana.baccigalupi@unina.it

5 School of Life Sciences, University of Lincoln, Lincoln LN6 7TS, UK; eferrari@lincoln.ac.uk

6 Department of Biology, Federico II University of Naples, 80126 Napoli, Italy; ericca@unina.it

7 Centro de Biología Molecular "Severo Ochoa" (CSIC-UAM), C. Nicolás Cabrera 1, Universidad Autónoma de Madrid, Canto Blanco, 28049 Madrid, Spain; mtrejas@cbm.csic.es (M.T.R.); wmeijer@cbm.csic.es (W.J.J.M.)

* Correspondence: s.cutting@rhul.ac.uk

check for

updates

Citation: Ferreira, W.T.; Hong, H.A.; Hess, M.; Adams, J.R.G.; Wood, H.; Bakun, K.; Tan, S.; Baccigalupi, L.;

Ferrari, E.; Brisson, A.; et al. Micellar Antibiotics of Bacillus. Pharmaceutics 2021, 13, 1296. https://doi.org/ 10.3390/pharmaceutics13081296

Academic Editors: Diego

Romano Perinelli and

Rita Muzzalupo

Received: 26 July 2021

Accepted: 12 August 2021

Published: 19 August 2021

Publisher's Note: MDPI stays neutral with regard to jurisdictional claims in published maps and institutional affiliations.

Copyright: (c) 2021 by the authors. Licensee MDPI, Basel, Switzerland. This article is an open access article distributed under the terms and conditions of the Creative Commons Attribution (CC BY) license (https:/ / creativecommons.org/licenses/by/ $4.0 /)$.
Abstract: Members of the Bacillus genus, particularly the "Bacillus subtilis group", are known to produce amphipathic lipopeptides with biosurfactant activity. This includes the surfactins, fengycins and iturins that have been associated with antibacterial, antifungal, and anti-viral properties. We have screened a large collection of Bacillus, isolated from human, animal, estuarine water and soil samples and found that the most potent lipopeptide producers are members of the species Bacillus velezensis. B. velezensis lipopeptides exhibited anti-bacterial activity which was localised on the surface of both vegetative cells and spores. Interestingly, lipopeptide micelles (6-10 nm diameter) were detectable in strains exhibiting the highest levels of activity. Micelles were stable (heat and gastric stable) and shown to entrap other antimicrobials produced by the host bacterium (exampled here was the dipeptide antibiotic chlorotetaine). Commercially acquired lipopeptides did not exhibit similar levels of inhibitory activity and we suspect that micelle formation may relate to the particular isomeric forms produced by individual bacteria. Using naturally produced micelle formulations we demonstrated that they could entrap antimicrobial compounds (e.g., clindamycin, vancomycin and resveratrol). Micellar incorporation of antibiotics increased activity. Bacillus is a prolific producer of antimicrobials, and this phenomenon could be exploited naturally to augment antimicrobial activity. From an applied perspective, the ability to readily produce Bacillus micelles and formulate with drugs enables a possible strategy for enhanced drug delivery.

Keywords: Bacillus velezensis; biosurfactant; micelle; antimicrobial; drug delivery; chlorotetaine

\section{Introduction}

Found typically in the saprophytic layers of soil Bacillus can produce endospores (spores) that survive indefinitely in a dormant form [1]. As spores, the bacterium can be disseminated easily in air and dust and form a major part of the aerobiome [2]. Consumption of plants, vegetables and other matter introduces spores into the gastrointestinal (GI)-tract where their intrinsic robustness enables passage across the gastric barrier. In the small intestine (jejunum and ileum), spores germinate and proliferate and in the large intestine they sporulate before being shed in the faeces [3]. In the GI tract, numbers of Bacillus are noticeably low $\left(\sim 10^{4} / \mathrm{g}\right.$ faeces in humans), but they are readily found in most animals suggesting that Bacillus is a minor allochthonous commensal [4]. Survival in dual 
communities (environment and intestinal) potentially presents an additional challenge to bacteria compared to those inhabiting a single niche. Production of antimicrobials plays an important part in competing with other bacteria and it is perhaps not surprising then, that members of the genus Bacillus are prolific producers of antimicrobials devoting $4-5 \%$ of its genome exclusively for this purpose $[5,6]$. Most of these compounds are peptides produced ribosomally (bacteriocins, enzymes) or non-ribosomally (lipopeptides, siderophores, polyketides) and are typically of low MW (5-10 kDa) [7,8]. In this work, we have screened Bacillus species isolated from diverse sources focusing on two phenotypic properties, antimicrobial activity and biosurfactant activity where the latter is typically indicative of the lipopeptide antibiotics (surfactin, fengycins and iturins [9]). We found that most strains exhibiting the highest levels of these dual activities belonged to one species, Bacillus velezensis. Using different pathogens including Clostridioides difficile we show that these Bacillus lipopeptides play an important role in antibacterial activity, first, by forming detergent-like mixed micelles and second, by incorporating antibiotics produced by Bacillus. The ability to incorporate lipopeptides and antibiotics into a micellar complex maximises antibacterial activity and is likely to have been adopted by this bacterium to its advantage.

\section{Materials and Methods}

\subsection{General Methods and Strains}

Strains screened for antimicrobial activity were presumed to be Bacillus due to the heat resistant step $\left(68{ }^{\circ} \mathrm{C}, 30 \mathrm{~min}\right)$ and aerobic culture used in their isolation. Isolates in our collection were previously recovered from human $(n=166)$, animal (cows $(n=137)$, pigs $(n=117)$, chickens $(n=139)$, estuarine water $(n=143)$ and soil samples $(n=132)$. A well diffusion assay was used to screen supernatants from Bacillus strains grown for $18 \mathrm{~h}$ in BHIB (brain heart infusion broth) at $37^{\circ} \mathrm{C}$. Using appropriate media, indicator strains were grown to the mid-logarithmic phase at $37^{\circ} \mathrm{C}$ and spread onto agar plates. Next, $50 \mu \mathrm{L}$ of Bacillus supernatant (filter-sterilised) was applied to pre-cut wells ( $5 \mathrm{~mm}$ diameter). After overnight incubation, zones of inhibition were measured. The resistance properties of culture supernatants were assessed using heat (oven, $90{ }^{\circ} \mathrm{C}, 15 \mathrm{~min}$ ), solvents, SDS $(0.1 \% v / v)$ and glutaraldehyde $(0.1 \% v / v)$ by mixing (1:1) and incubation at RT with periodic vortexing. Pathogen growth kinetic experiments were conducted using mid-logarithmically growing cultures of the target strain, dividing the culture into two and to one flask adding 1:10 volume of a cell-free supernatant of the relevant Bacillus strain. C. difficile (CD630) was routinely grown in BHIS medium (brain heart infusion supplemented with yeast extract and cysteine) agar ( $37 \mathrm{~g}$ brain heart infusion, $5 \mathrm{~g}$ yeast extract and $1 \mathrm{~g}$ L-cysteine per litre). For determining the minimum inhibitory concentration (MIC) of Bacillus lipopeptides, the CD630 culture was grown in BHIS until the $\mathrm{OD}_{600}$ reached $\sim 0.2$, at which point SG277 SEC purified lipopeptides were added at different 2 -fold concentrations, in the range $1000-0.5 \mu \mathrm{g} / \mathrm{mL}$ (as estimated by dry weight). The cultures were left to incubate anaerobically for $18 \mathrm{~h}\left(37^{\circ} \mathrm{C}\right)$. The $\mathrm{OD}_{600}$ of the cultures were measured and the MIC was determined, with the endpoint being defined as the lowest concentration where the OD $<50 \%$ of the CD630 "media only" control.

\subsection{Biosurfactant Activity}

Biosurfactant activities of Bacillus supernatants were determined using an oil-displacement method using sunflower oil [10].

\subsection{Microdilution Assay of $C$. difficile Inhibitory Activity}

Plates were set up as follows, $216 \mu \mathrm{L}$ of sterile BHIS was pipetted into the first row of a 96-well U-bottom microplate (Greiner Bio-One, Gloucestershire, UK) and $120 \mu \mathrm{L}$ into each subsequent row. Next, $24 \mu \mathrm{L}$ of the sample to be tested was pipetted into the first row (dilution factor of 1:10) and serially diluted in a 2-fold dilution series until the last row (1:1280 dilution factor) on the microplate. A media-only control was also applied into a single well of the first column and serially diluted. Subsequently, $12 \mu \mathrm{L}$ of a $6 \mathrm{~h}$ 
CD630 indicator culture was pipetted into each well and the plate was incubated for $18 \mathrm{~h}$ at $37^{\circ} \mathrm{C}$ in an anaerobic chamber. After incubation, the microplate contents were agitated on a rotary plate shaker at $200 \mathrm{rpm}$ for $2 \mathrm{~min}$ after which the $\mathrm{OD}_{600}$ was measured using a microplate plate reader. Positive inhibitory activity was defined as an $\mathrm{OD}_{600}<50 \%$ of the CD630 "media only" control.

\subsection{Purification Procedures}

For ammonium sulphate $\left(\mathrm{AmSO}_{4}\right)$ precipitation, filter-sterile supernatant was precipitated overnight with $\mathrm{AmSO}_{4}\left(4{ }^{\circ} \mathrm{C}\right)$ using saturated $\mathrm{AmSO}_{4}$ (Sigma-Aldrich, Dorset, UK) (Supplementary Figure S3) solution at a final concentration of 20\% $(w / v)$. Following centrifugation at $10,000 \times g$ for $15 \mathrm{~min}$, the supernatant was removed, and the precipitate was resuspended in PBS (Sigma-Aldrich, Dorset, UK). To remove excess $\mathrm{AmSO}_{4}$ the filtrate was dialyzed using 3500 daltons MEMBRA-CEL dialysis tubing (Viskase, Chesterfield, UK) against PBS overnight at $4{ }^{\circ} \mathrm{C}$.

For size-exclusion chromatography (SEC) analysis, $1.5 \mathrm{~mL}$ of PBS was added to $1 \mathrm{~mL}$ of the dialysed $\mathrm{AmSO}_{4}$ precipitate and $2.5 \mathrm{~mL}$ applied to a Superdex 200 column $(\mathrm{L} \times$ I.D. $30 \mathrm{~cm} \times 10 \mathrm{~mm})$ and fractionated by SEC with PBS as the running buffer. Fractions were tested for activity against CD630 using the microdilution assay and positive fractions combined, dialyzed against PBS overnight at $4{ }^{\circ} \mathrm{C}$ and concentrated using a $10 \mathrm{kDa}$ Vivaspin (Sartorius, St. Neots, UK) MWCO centrifugal concentrator to a final volume of $1 \mathrm{~mL}$. Henceforth, this is referred to as the active SEC fraction.

The SEC "active" sample was separated by RP-HPLC using uBondapack Phenyl, $125 \AA$, $10 \mu \mathrm{m}, 3.9 \times 300 \mathrm{~mm}$ (Waters, Manchester, UK) column and Waters 600 Multisolvent Delivery system, comprising Waters 600E System Controller and Waters 600 Fluid Unit, and complemented with an ABI 757 Spectroflow absorbance detector (Kratos, Manchester, UK). The mobile phase components were Buffer A $(0.5 \%(v / v)$ acetic acid in $60 \%(v / v) \mathrm{MeOH})$ and Buffer $\mathrm{B}(0.5 \%(v / v)$ acetic acid in $95 \%(v / v) \mathrm{MeOH})$. The fractions were injected in Buffer $\mathrm{A}$ and the products eluted (flow rate $0.5 \mathrm{~mL} \mathrm{~min}^{-1}$ ) with a linear gradient of Buffer $\mathrm{B}$, developed from $0 \%$ to $100 \%$ ( $60 \mathrm{~min}$ ). The elution pattern was monitored by determining absorbance at $220 \mathrm{~nm}$, and resultant fractions were concentrated using an EZ-2 Genevac centrifugal evaporator (Genevac Ltd., Ipswich, UK) and then either tested for inhibitory activity against $\mathrm{CD} 630$ (by resuspension in $\mathrm{dH}_{2} \mathrm{O}$ ) using a microdilution assay or identified by mass spectrometry (MALDI-TOF).

MALDI-TOF-MS analysis was performed on a Bruker Reflex III system (Bruker Daltonics, Billerica, MA, USA) equipped with a sealed nitrogen pulsed laser ( $337 \mathrm{~nm}$, line-width $0.1 \mathrm{~nm}, 4 \mathrm{~ns}$ pulse duration, 300 microjoules rated pulse energy, average power $5 \mathrm{~mW}$ at $200 \mathrm{~Hz}$ ). A working solution of matrix was prepared by mixing $60 \mu \mathrm{L}$ of the alpha-cyano4-hydroxycinnamic acid matrix stock solution in $\mathrm{MeOH}$ (MALDI-QUALITYTM , Agilent Technologies, Santa Clara, CA, USA), $30 \mu \mathrm{L}$ of acetone and $1 \mu \mathrm{L}$ of $1 \%(v / v)$ TFA in a glass vessel. Individual RP-HPLC fractions were mixed with the matrix solution and deposited onto a stainless steel MALDI plate. Mass spectra were acquired using the reflection mode. The instrument was calibrated to a mass accuracy of at least $30 \mathrm{ppm}$ using C18 purified sets of proteolytic albumin peptides to cover the range of masses from ca. $500 \mathrm{~m} / z$ to ca. $2000 \mathrm{~m} / z$ ) and using $\alpha$-cyano-4-hydroxycinnamic acid (CHCA, monoisotopic mass 189.0426) and CHCA clusters to map the lower MW region of ca. $200 \mathrm{~m} / z$ to ca. $800 \mathrm{~m} / \mathrm{z}$. In addition to extensive MALDI-TOF-MS analyses, masses were also confirmed using Matrix-Less Nanostructured Assisted Laser Desorption Ionisation TOF-MS (NALDI-TOFMS) using a Bruker Reflex III system. In all cases, the TOF-MS analysis was conducted in reflector mode and 500 laser shots were accumulated for each mass spectrum.

\subsection{Dynamic Light Scattering (DLS) Analysis}

Active SEC material (in $150 \mathrm{mM}$ sodium phosphate buffer, $\mathrm{pH}$ 7.2) was cleared by centrifugation at $17,000 \times g$ for $1 \mathrm{~h}$ prior to analysis. Next, $100 \mu \mathrm{L}$ of sample was transferred to a micro-cuvette, equilibrated at $25{ }^{\circ} \mathrm{C}$ and measured in triplicate using a Zetasizer 
Nano ZS (Malvern Panalytical, London, UK). Particle diameter was estimated from the Z-average size. Polydispersity index (PDI) and cumulant fit intercept (CFI) were obtained from cumulants fit analysis using Zetasizer software v7.11 (Malvern Panalytical, London, UK). Data points are the average of three measurements with error bars representing the standard error.

\subsection{Bactericidal Activity of SG277 Micellar Material}

B. velezensis SG277 (a human isolate, SporeGen Ltd., London, UK) was grown in $\mathrm{BHIB}$ for $18 \mathrm{~h}$ at $37^{\circ} \mathrm{C}$ resulting in $>90 \%$ spore formation. The culture was centrifuged, and the supernatant was retained. The pellet from $1 \mathrm{~mL}$ of culture $\left(\sim 5 \times 10^{8} \mathrm{CFU}\right)$ was washed 3-times with PBS and resuspended in $1 \mathrm{~mL}$ of a mid-logarithmic stage CD630 culture. Cultures were gently agitated to prevent sedimentation of Bacillus spores. The collected supernatant was added to the same culture at a dilution factor of $1 / 10(v / v)$, $\mathrm{AmSO}_{4}$ precipitate at 1/320 (v/v), SEC active fraction at 1/160 (v/v) and the RP-HPLC purified surfactin at $1 / 20(v / v)$. The dilutions were determined according to normalisation of activity as measured in a previously performed microdilution assay. $\mathrm{dH}_{2} \mathrm{O}$ was added to the CD630 culture as a negative control. Samples were taken hourly, serially diluted and plated on BHISS agar plates and incubated for $24 \mathrm{~h}$ at $37^{\circ} \mathrm{C}$ at which point colonies were enumerated and CFU/mL determined.

\subsection{Microfiltration Experiments}

SG277 was grown for $18 \mathrm{~h}$ in BHIB and the culture supernatant was centrifuged two-times $(8000 \times g, 15 \mathrm{~min}, \mathrm{RT})$. The salt concentration of the supernatant was adjusted to $\sim 150 \mathrm{mM}$ and $\mathrm{pH} 7.0$ by adding $\mathrm{NaH}_{2} \mathrm{PO}_{4} \mathrm{H}_{2} \mathrm{O}(5 \% \mathrm{w} / v)$ followed by centrifugation $(21,000 \times g, 3 \mathrm{~h}, \mathrm{RT})$ to remove any aggregates. The cleared supernatant was then filtered through a series of syringe filters and 100, 30, 10 and $5 \mathrm{kDa}$ Vivapsin (Sartorius, St. Neots, UK) MWCO cut-off spin columns $(6000 \times g$ for $10 \mathrm{~min})$. Each filter was first equilibrated with sterile BHIB medium before use. After filtration, eluates were collected, and membranes were washed with $1 \mathrm{~mL}$ of methanol (100\%) in order to extract entrapped material. Eluates and the methanol filtered wash were checked for activity against CD630 using a microdilution inhibition assay. The outcome of the assay was registered as the lowest dilution at which the growth of CD630 was suppressed.

\subsection{Agar Plate Inhibition Assay}

SG277 was grown in BHIB for $18 \mathrm{~h}$ at $37^{\circ} \mathrm{C}$ resulting in $>90 \%$ spores (data not shown). The culture was centrifuged, and the supernatant retained. The pellet was washed 3 times with PBS. Both pellet (suspended in PBS) and retained supernatant were heat treated $\left(80{ }^{\circ} \mathrm{C}, 15 \mathrm{~min}\right)$ to kill residual cells. Resuspended pellet or supernatant was mixed in equal volume $(0.25 \mathrm{~mL})$ with a logarithmic culture of CD630. This was then added to $4.5 \mathrm{~mL}$ of $0.6 \%$ micropropagation agar (Apollo Scientific, Manchester, UK) and poured on BHIS agar plates and incubated anaerobically overnight at $37^{\circ} \mathrm{C}$. Inhibition was apparent compared against the turbid appearance of untreated CD630.

\subsection{Combination of Micelles and Antibiotics}

The lipopeptide content of $30 \times \mathrm{AmSO}_{4}$ (see above) was estimated to be $\sim 10 \mathrm{mg} / \mathrm{mL}$. The $30 \times \mathrm{AmSO}_{4}$ was dried with a Genevac EZ-2 SpeedVac evaporator (Genevac Ltd., Ipswich, UK) and the material resuspended in methanol to a final concentration of $\sim 0.2 \mathrm{mg} / \mathrm{mL}$. This solution was then mixed with stock solutions of vancomycin $(0.2 \mathrm{mg} / \mathrm{mL})$, clindamycin $(0.2 \mathrm{mg} / \mathrm{mL})$ or metronidazole $(1 \mathrm{mg} / \mathrm{mL})$ (Sigma-Aldrich, Dorset, UK), to achieve a 1:1 micelle:antibiotic ratio. Methanol was removed from the mixture using a SpeedVac and the pellet was suspended in $1 \mathrm{~mL}$ of sterile $1 \times$ PBS ( $\mathrm{pH} 7.4$ ), bringing the concentration of micelles to $\sim 1 \mathrm{mg} / \mathrm{mL}$. The solutions were vortexed (15 min, RT) and then centrifuged $(4000 \times g)$ in a tabletop centrifuge at $37^{\circ} \mathrm{C}$ for $3 \mathrm{~h}$ and kept overnight at $4^{\circ} \mathrm{C}$. After overnight storage, the solutions were centrifuged $(1 \mathrm{~h}, 4000 \times g, \mathrm{RT})$ and filtered through a $1.2 \mu \mathrm{m}$ 
filter in order to remove any potential aggregates. The flow-through was then filtered again through a $10 \mathrm{kDa}$ Vivaspin MWCO $(4000 \times g, 1.5 \mathrm{~h})$. The starting solution, retentate $(\mathrm{MeOH}$ wash of the membrane) and flow-through were equilibrated to the same volume and tested against $630 \Delta e r m$ (an erythromycin-resistant derivative of CD630) using a microdilution inhibition assay.

\subsection{Micellar Lipopeptide Interactions with Resveratrol}

A total of $50 \mu \mathrm{g}$ of resveratrol (RSV) was mixed with $500 \mu \mathrm{L}$ of SG277 SEC purified material (containing $\sim 0.5 \mathrm{mg}$ of lipopeptides) or PBS only (pH 7.4) and incubated while shaking for $18 \mathrm{~h}$ at RT. The mixture was centrifuged $(21,000 \times g, 20 \mathrm{~min})$, the supernatant collected and vacuum-evaporated until dry. The pellets were resuspended in $\mathrm{MeOH}$, and resveratrol was detected and quantified against standards using RP-HPLC as described above, with absorbance at $295 \mathrm{~nm}$ determined. Aqueous solubility of resveratrol was calculated as a percentage of the solubilised quantity in the PBS only sample. For resveratrol micellar incorporation experiments, the above-collected supernatant was applied to a $10 \mathrm{kDa}$ MWCO membrane, centrifuged $(6000 \times g, 2 \mathrm{~h})$ and the resulting eluate $(<10 \mathrm{kDa})$ was removed. The retentate $(>10 \mathrm{kDa})$, including a $\mathrm{MeOH}$ membrane wash, was also collected. The eluate and retentate solutions were vacuum evaporated until dry, resuspended in proportional volumes of $\mathrm{MeOH}$, and resveratrol was detected and quantified against standards using RP-HPLC. The proportion of resveratrol in the eluate or retentate was calculated as a proportion of the total amount.

\subsection{Cryogenic Electron Microscopy (Cryo-EM)}

A $4 \mu \mathrm{L}$ aliquot was placed onto an EM grid coated with a perforated carbon film. Excess liquid was removed with filter paper, and grids were plunge-frozen into liquid ethane cooled by liquid nitrogen using a Leica EMCPC cryo-chamber (Leica Microsystems, Milton Keynes, UK). For Cryo-EM observation, grids were mounted onto a Gatan 626 cryoholder (Gatan Inc., Pleasanton, CA, USA) and transferred to a Tecnai F20 microscope (ThermoFisher, Waltham, MA, USA) operated at $200 \mathrm{kV}$. Images were captured with an Eagle 2k CCD camera (FEI, Hillsboro, OR, USA).

\subsection{Visualization by Transmission Electron Microscopy (TEM)}

Samples (concentrated $\mathrm{AmSO}_{4}$ precipitated material) were adsorbed for 3 min to glowdischarged collodion/carbon-coated copper grids. Grids were then washed twice with bidistilled water, negatively stained for $50 \mathrm{~s}$ with $2 \%$ uranyl acetate in water and air-dried before visualization under a JEM1010 transmission electron microscope (JEOL, Tokyo, Japan). Images were acquired with a TVIPS F416 CMOS camera (TVIPS, Gauting, Germany).

\subsection{Biofilms}

Biofilms were prepared on either DSM or S7 medium as described [11]. For SEM analysis, aluminium foils were placed into the wells of 12 -well plates. The bacterial cultures were added (at an OD600 of 0.1) and incubated at $37^{\circ} \mathrm{C}$ for $48-72 \mathrm{~h}$ under static growth conditions. After culturing, the biofilms were fixed with $2.5 \%$ glutaraldehyde $(v / v)$ overnight at $5{ }^{\circ} \mathrm{C}$, post-fixed with $1 \%$ osmium tetroxide $(w / v)$ for $1.5 \mathrm{~h}$ at RT, dehydrated with ethanol, air-dried overnight and finally mounted on aluminium stubs. Samples were then washed with PBS, fixed and dehydrated. The dehydrated samples were coated with a gold layer and observed under a Scanning Electron Microscope (SEM) (FEI QUANTA 200, FEI company, Hillsboro, OR, USA) in high vacuum mode.

\section{Results}

\subsection{B. velezensis Is a Prolific Producer of Antimicrobial Activity}

Cell-free supernatants from over 800 Bacillus strains isolated from a variety of sources were screened for antimicrobial activity against a panel of Gram-positive (Staphylococcus aureus, Staphylococcus hominis, Bacillus cereus, C. difficile and Listeria monocytogenes) and 
Gram-negative (Escherichia coli, Salmonella enterica and Klebsiella aerogenes) pathogens using a well-diffusion assay. Approximately $80 \%$ of strains showed some level of inhibitory activity to at least one pathogen. Thirty-six strains that exhibited the highest levels of inhibitory activity were shortlisted and their species assigned using analysis of the gyr $A$ and/or 16S rRNA genes (Supplementary Table S2). In each case, extracellular inhibitory activity was found to be resistant to heat $\left(90^{\circ} \mathrm{C}, 15 \mathrm{~min}\right.$ ) and apart from one (SG836) all strains carried strong biosurfactant activity in the cell-free culture supernatants. Except for four strains (SG215, SG655, SG836 and SG224), the remainder $(n=32)$ belonged to the "B. subtilis group" [12]. Most interesting was that $\sim 80 \%$ of the isolated strains were $B$. velezensis $(n=24)$ or its close relative B. amyloliquefaciens $(n=2)$, which are known to typically exhibit biosurfactant properties due to the synthesis of lipopeptides including the surfactins, fengycins and iturins $[13,14]$.

Further analysis revealed that the nature of antibacterial activity varied considerably between strains with bacteriostatic, bactericidal as well as bacteriolytic activity (Figure 1, Supplementary Figure S1 and Supplementary Table S2). Bacteriostatic activity was observed against all Gram-negative pathogens while a broader range of activities (bacteriostatic, bactericidal or bacteriolytic) was evident against Gram-positives.

A S. enterica (SG576)

B E. coli 0157:H7 (SG588)

C C. difficile (CD630)
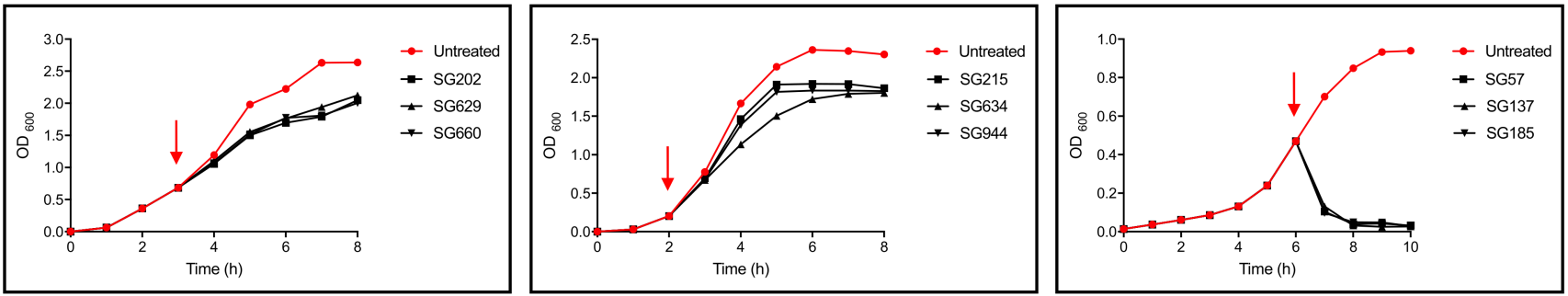

D

S. aureus (SG548)

E L. monocytogenes (NCTC 19115)

F $\quad$ L. monocytogenes (NCTC 19115)
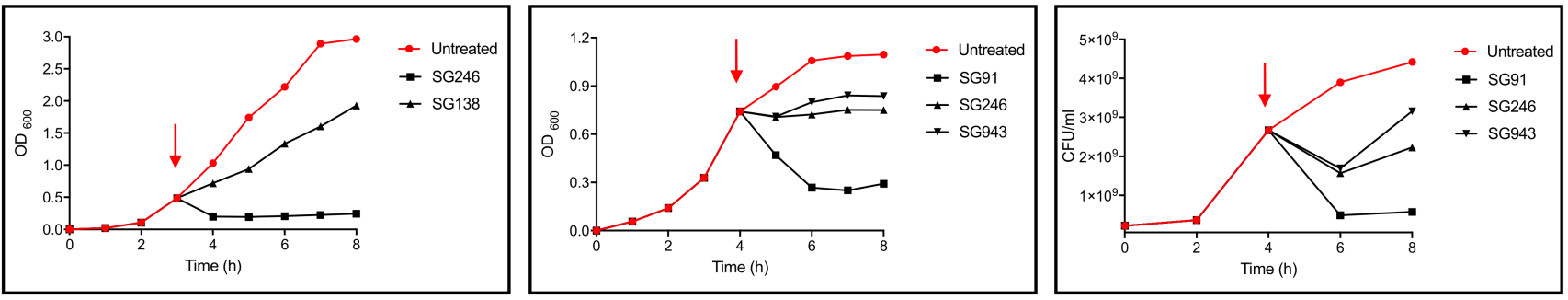

Figure 1. Pathogen growth kinetics in the presence of active components. Cell-free supernatants from Bacillus strain cultures (SGx) were added (1:10 v/v, indicated by red arrow) to mid-logarithmic cultures of Gram-negative and Gram-positive pathogens (A-F). (A) SG202, SG629 and SG660 supernatant was added (at time indicated by red arrow) to S. enterica (SG576); (B) SG215, SG634 and SG944 supernatant was added (at time indicated by red arrow) to E. coli 0157:H7 (SG588), (C) SG57, SG137 and SG185 supernatant was added (at time indicated by red arrow) to C. difficile (CD630); (D) SG246 and SG138 supernatant was added (at time indicated by red arrow) to S. aureus (SG548); (E) SG91, SG246 and SG943 supernatant was added (at time indicated by red arrow) to L. monocytogenes (NCTC 19115); (F) SG91, SG246 and SG943 supernatant was added (at time indicated by red arrow) to L. monocytogenes (NCTC 19115). Growth $\left(\mathrm{OD}_{600}\right)$ was monitored in cultures with or without (untreated) addition of supernatants. For L. monocytogenes growth by $\mathrm{OD}_{600}$ and by determination of CFU/mL.

\subsection{Characterisation of Inhibitory Activity}

The abundance of $B$. velezensis strains with both heat-resistant inhibitory activity and biosurfactant properties might indicate a common mechanism of action. We focused on one pathogen, $C$. difficile strain 630 (CD630; ribotype 012 , tox $\mathrm{A}^{+}$toxB ${ }^{+}$), and the most potent B. velezensis strains (SG57, SG137, SG185, SG277 and SG297) for further analysis using a microdilution assay to quantify inhibitory activity. All strains were genome sequenced and 
confirmed as B. velezensis having ANI (average nucleotide identity) scores of $>95 \%$ when compared to B. velezensis type strain ATR2 (Supplementary Table S1). SG277 and SG297 had inhibitory activities of 1:160 against CD630 while the remaining three strains had activities of 1:80. Activity in the culture supernatants exhibited complete resistance to heat $\left(100{ }^{\circ} \mathrm{C}\right.$, $30 \mathrm{~min})$, solvents (toluene, acetone and chloroform), SDS $(0.1 \% v / v)$ and glutaraldehyde $(1 \% v / v)$. Using centrifugal concentrators of different molecular weight cut-offs (MWCO) we determined that the antibacterial activity present in SG277 cell-free supernatants was present in two fractions, $30-100 \mathrm{kDa}$ and $>100 \mathrm{kDa}$ suggesting the presence of a partially labile complex. In addition, we verified that equivalent levels of inhibition were present against our collection of over thirty $C$. difficile ribotypes (rt) including those known to be hypervirulent (rt 027) and zoonotic (rt 078) (data not shown). Ammonium sulphate $\left(\mathrm{AmSO}_{4}\right)$ was also used for the precipitation of active material in the cell-free supernatant. Empirically, we found that a $20 \%(w / v)$ cut precipitated material that retained both inhibitory activity against CD630 (activity of 1:2560 in a 30× concentrate as measured using a microdilution assay) and biosurfactant activity. Transmission electron microscopy (TEM) of this precipitated material again revealed the presence of large granular aggregates of approximately 20-50 $\mathrm{nm}$ in size (Figure 2A). SDS-PAGE analysis of the $\mathrm{AmSO}_{4}$ precipitated material revealed a low MW band of $\sim 1 \mathrm{kDa}$ that was weakly stained with Coomassie blue and Alcian blue but not with oil-red (Figure 2B). Interestingly, a band of equal size and migration to a similar position was also apparent in "unstained" gels as a white species (labelled WB in Figure 2B). When the unstained gel lane was excised, SDS eluted and then overlaid with soft agar containing CD630 cells, following overnight anaerobic incubation, growth was inhibited in the region corresponding to the white band after overnight anaerobic incubation of the plate (Figure 2C). The $\mathrm{AmSO}_{4}$-precipitated active material was further purified by size exclusion chromatography (SEC) and reversed-phase HPLC (RP-HPLC). Cryo-EM analysis of SEC-purified material that retained inhibitory activity against CD630 revealed an abundance of homogenous structures resembling circular micelles with a diameter of $\sim 6-10 \mathrm{~nm}$ (Figure 2D). DLS analysis confirmed that the material was homogeneous with most particles presenting a size within the $6-10 \mathrm{~nm}$ range, together with a minority population having a size of 150-200 nm (Figure 2E). Size distribution of these particles in sodium phosphate buffer ( $\mathrm{pH}$ 7.2) was stable over several hours, with the only change being a reduction in aggregates larger than $100 \mathrm{~nm}$ indicating sedimentation.

A

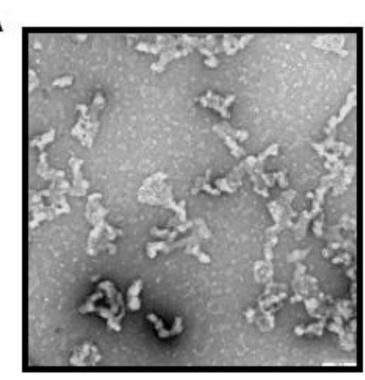

D

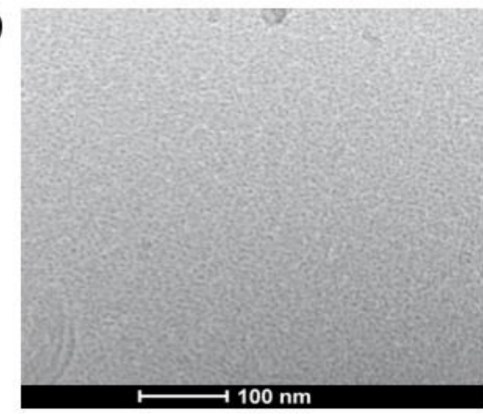

B

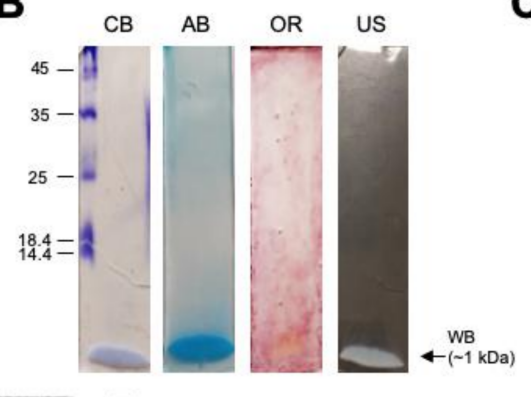

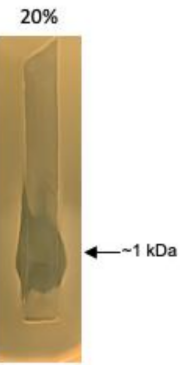

E

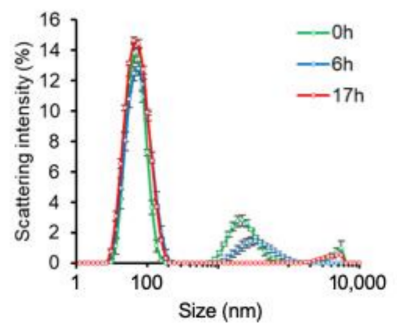

Figure 2. Activity corresponds to low MW species. (A) TEM analysis of $\mathrm{AmSO}_{4}$ precipitated material $(20 \%)$, scale bar $=100 \mathrm{~nm}$. (B) Staining of the $20 \% \mathrm{AmSO}_{4}$ material run on $12.5 \%$ SDS-PAGE gels 
showed staining with Coomassie blue $(\mathrm{CB})$ and Alcian blue $(\mathrm{AB})$ but no detectable staining with oil-red (OR). Unstained gels (US) also showed clearly a $1 \mathrm{kDa}$ unstained white band (WB). (C) The unstained gel lane was excised, SDS-eluted and the lane placed on BHIS agar and then overlaid with BHIS soft $(0.6 \%)$ agar containing live CD630 cells. Following overnight growth under anaerobic conditions only the region corresponding to the $1 \mathrm{kDa}(\mathrm{WB})$ band showed inhibition of CD630 growth. (D) Cryo-EM analysis of SEC material. The majority of the field is occupied with small $(<10 \mathrm{~nm})$ micelles appearing as granular-like objects. (E) DLS analysis of size distribution of the SG277 SEC fraction taken at different times following dilution in $150 \mathrm{mM}$ sodium phosphate buffer at $\mathrm{pH} 7.2$ shows the stability of the micelles over $17 \mathrm{~h}$. Data points are the average of three measurements with error bars representing the standard error.

\subsection{Inhibitory Activity Is Due to the Formation of Mixed Micellar Complexes}

SEC-purified material was separated by RP-HPLC, after which the individually collected fractions were tested for inhibitory activity against CD630 (Figure 3) and analysed by MALDI-TOF (Table 1). For active fractions derived from SG277 (and SG297) three classes of lipopeptide, surfactins, fengycins and iturins (iturin A and mycosubtilin) were readily detectable together with a dipeptide antibiotic chlorotetaine (MW 300 Da; [15]). SEC purified material was determined to have an MIC of $4 \mu \mathrm{g} / \mathrm{mL}$ against C. difficile. Strains SG277 and SG297, which exhibited the highest levels of inhibitory activity against CD630, produced chlorotetaine, iturins, fengycins and surfactins. Strains that displayed lower inhibitory CD630 activity did not produce chlorotetaine (SG57, SG137 and SG185) or chlorotetaine and iturins (SG137 and SG185) (data not shown). Our analysis also revealed trace amounts of glycolipids including rhamnolipids and sophorolipids (data not shown) which may account for the Alcian blue staining (Figure 2B). However, we cannot rule out the possibility that this might also have arisen by contamination from the biofilm matrix (see below) which is typically rich in polyamines [16].

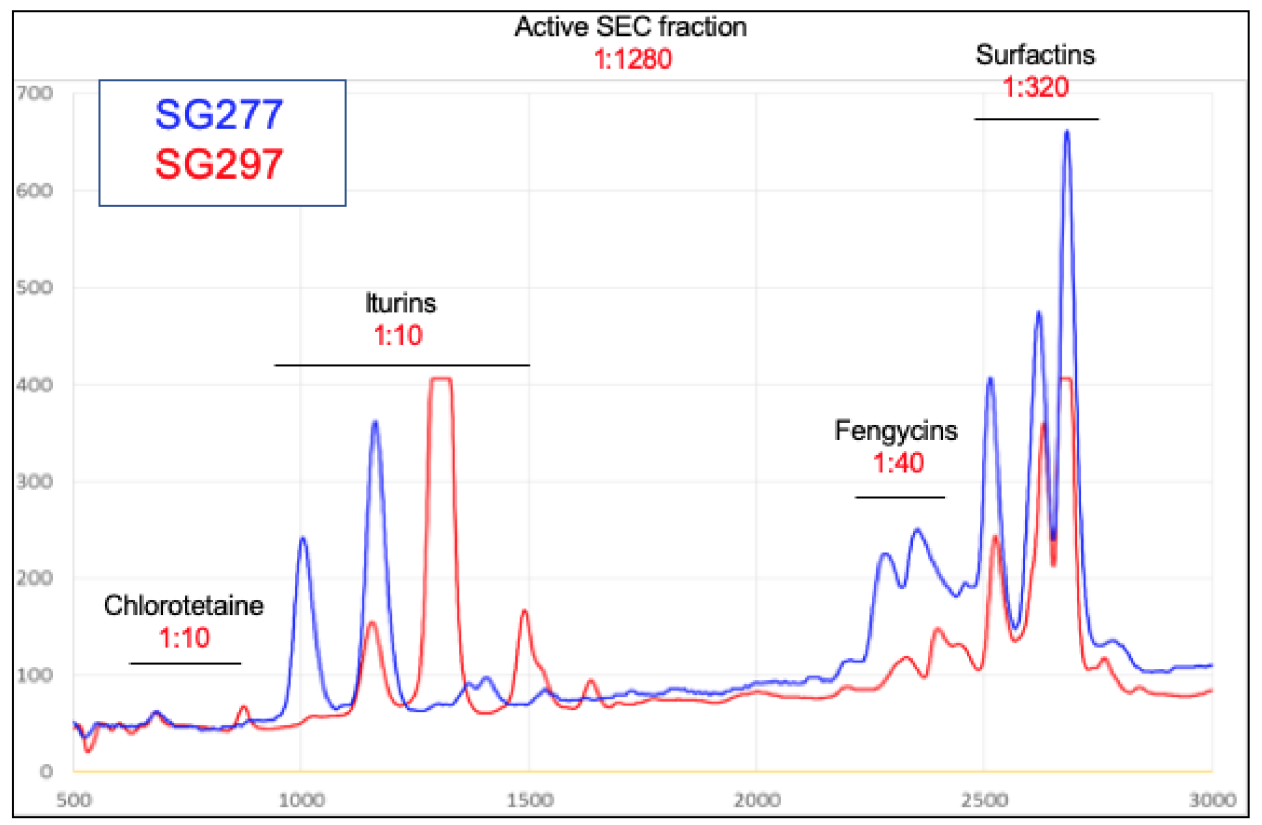

Figure 3. RP-HPLC separation and MALDI-TOF analysis of active components. RP-HPLC separation of the active SEC fraction from SG277 and SG297 using a gradient of $65-95 \% \mathrm{MeOH}(+0.1 \%$ acetic acid). Peaks were analysed using MALDI-TOF and compounds are indicated in the figure. Initial inhibitory activity of the SEC fraction was 1:1280 and those in RP-HPLC fractions are indicated in red type. 
Table 1. Components identified during MALDI-TOF analysis for peaks collected during RP-HPLC separation of the SG277 active SEC fraction.

\begin{tabular}{cc}
\hline Compounds & SG277/SG297 Identities \\
\hline Chlorotetaine & $\begin{array}{c}\text { Chlorotetaine }\left({ }^{35} \mathrm{Cl}\right)\left({ }^{37} \mathrm{Cl}\right) \\
\text { Hydroxychlorotetaine }\left({ }^{35} \mathrm{Cl}\right)\left({ }^{37} \mathrm{Cl}\right)\end{array}$ \\
\hline \multirow{2}{*}{ Iturins } & $\mathrm{C}_{12-17}$ Iturin \\
& $\mathrm{C}_{11-17}$ Bacillomycin F \\
& $\mathrm{C}_{11-17}$ Mycosubtilin \\
\hline \multirow{2}{*}{ Fengycins } & $\mathrm{C}_{15-19}$ Fengycin A \\
& $\mathrm{C}_{13-17}$ Fengycin B \\
& $\mathrm{C}_{17}$ Fengycin C \\
& $\mathrm{C}_{16}$ Fengycin D \\
& $\mathrm{C}_{17}$ Fengycin E \\
\hline \multirow{2}{*}{ Surfactins } & $\mathrm{C}_{12-17}$ Surfactin A \\
& $\mathrm{C}_{13-18}$ Surfactin B \\
& $\mathrm{C}_{12-17}$ Surfactin C \\
\hline
\end{tabular}

Using commercially acquired samples of surfactin (MW $\sim 1 \mathrm{kDa}$ ), iturins (MW $\sim 1 \mathrm{kDa}$ ) and fengycins (MW $\sim 1.5 \mathrm{kDa}$ ) we were unable to demonstrate any significant level of inhibitory activity to CD630 even when all three lipopeptides were combined. It has been previously reported that surfactin C-15 (15 carbon fatty acid chain) is the homologue most active against cell membranes where the higher number of carbon atoms in the fatty acid chain in conjunction with the single negative charge in the peptide ring results in a more active compound $[17,18]$. Interestingly, contrary to the low proportion of surfactin C-15 present in the commercially acquired surfactin sample (Supplementary Figure S2A), samples prepared from the Bacillus strain contained higher levels of surfactin C-15 (Supplementary Figure S2D). In particular, a high level of inhibitory activity was observed only in the SG277 RP-HPLC fraction containing proportionally higher amounts of C-15 surfactin (Supplementary Figure S2B-D).

We next prepared a cell-free supernatant from an SG277 culture and passed this through a series of microfiltration membranes in a stepwise process. Starting with a $1.2 \mu \mathrm{M}$ membrane, the supernatant was passed through and the activity in the eluate, as well as that retained on the filter, were determined (Figure 4). The eluate was then passed through further filters, at each step reducing the pore size, culminating in a $5 \mathrm{kDa}$ MWCO membrane. This analysis showed that the $100 \mathrm{kDa}$ MWCO membrane failed to allow passage of most of the inhibitory activity, with the remaining activity being retained on the subsequent $30 \mathrm{kDa}$ filter. The $100 \mathrm{kDa}$ MWCO membrane has a pore size of approximately $10 \mathrm{~nm}$ which is in close agreement with the predicted size of the SG277 micelles (Figure 2D-E) indicating the micelle size to be $\sim 100 \mathrm{kDa}$. Some activity was retained using higher MWCO membranes, but we attribute this to the presence of larger aggregates that are apparent from DLS analysis (Figure 2D). This shows then that inhibitory activity is more likely associated with the micellar form rather than the individual components (all of which have MWs of $<1.5 \mathrm{kDa}$ ). This is further supported by the high amount of anti-C. difficile activity observed when components are incorporated into mixed micelles (in the active SEC fraction), as opposed to the much lower levels of activity of individual components post RP-HPLC separation (Figure 3). 

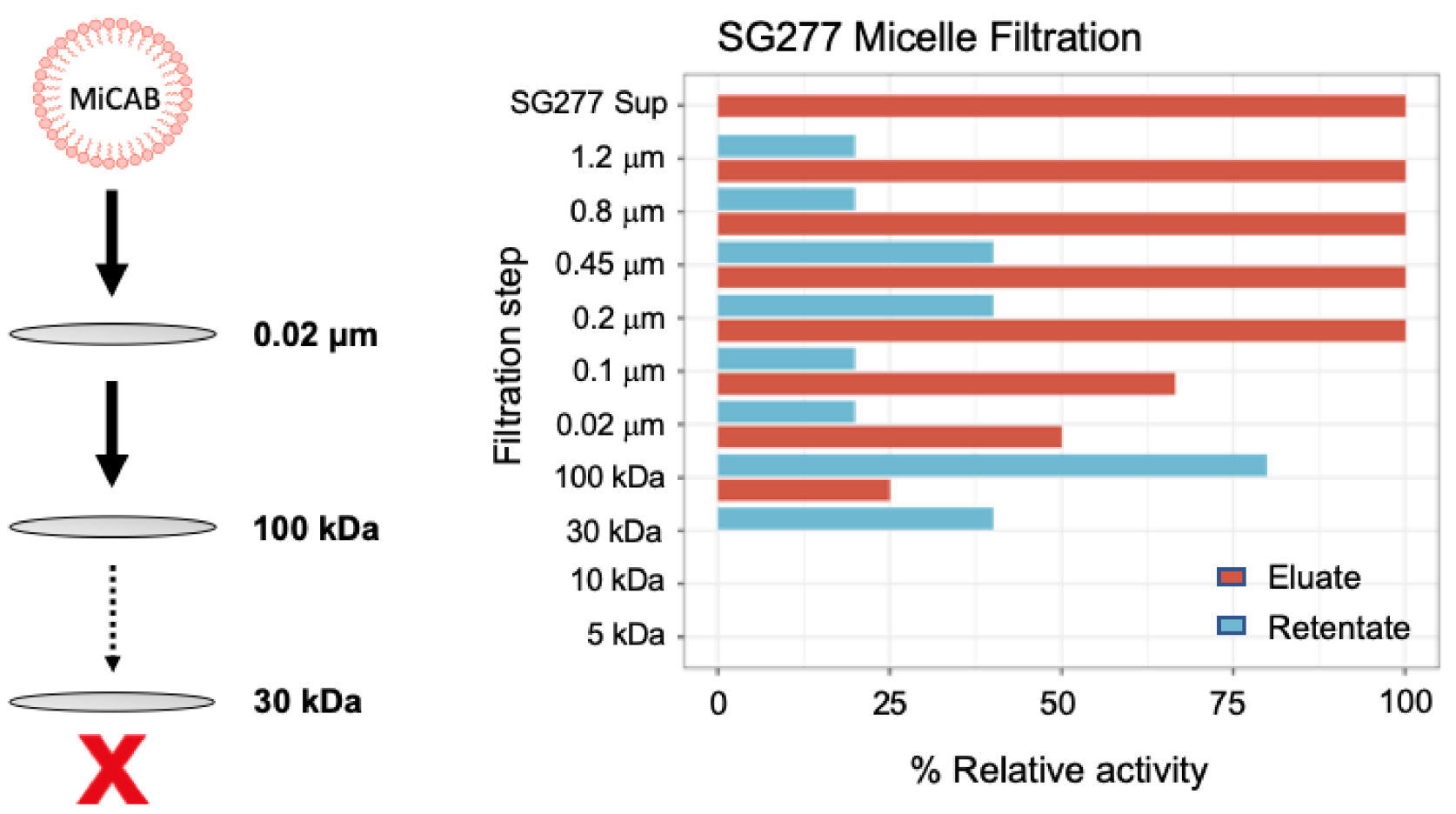

Figure 4. Activity is associated with micelles. Schematic diagram showing the Bacillus micelles and the step-wise filtration steps. The graph shows the resulting anti-CD630 activity in the eluate and retentate following filtration of an SG277 culture supernatant through a series of filters starting with $1.2 \mu \mathrm{m}$ and ending with a $5 \mathrm{kDa}$ MWCO membrane. Inhibitory activity to CD630 was determined using a microdilution assay and the data is the mean of 4 independent studies.

\subsection{Genome Analysis}

In strains with the highest activity against $C$. difficile strain CD630, surfactins, fengycins and iturins (both iturin A and mycosubtilin) were detectable by MS varying only by the length of their carbon chain. Trace levels of the lipopeptides Kurstakin [19] and Mojavensin A [20] were also detected. For strains with lesser activity (SG57, SG137 and SG185) only surfactins and fengycins were detectable by MS yet genomic analysis did reveal complete biosynthetic operons ( $f e n F$ and $m y c A-C)$. Operons responsible for surfactins $(\operatorname{srf} A A-D)$ and fengycins ( $y n g L, f e n A-E)$ were present although in the case of the fengycins the order of cistrons within the operon did differ between strains (Supplementary Table S1). All strains carried three clusters of polyketide synthase genes common to B. amyloliquefaciens and responsible for the synthesis of difficidin (and its oxidised form oxydifficidin), bacillaene and macrolactins $[5,21,22]$. As indicated by the name, difficidin is a polyketide with activity against a number of bacteria including $C$. difficile [23] and although we were able to identify this antibiotic in SEC-purified material it was not present in the "active" RP-HPLC fractions. No gene or genes encoding chlorotetaine could be found but all strains carried an operon of five genes responsible for the closely related bacilysin (a dipeptide antibiotic commonly found in members of the B. subtilis group $[24,25])$.

\subsection{Inhibitory Activity Associates with the Surface Layers of the Cell and Spore}

B. velezensis strains are, similar to those of B. amyloliquefaciens, highly mucoid resulting from the production of exopolysaccharides from the vegetative cell. We demonstrated that strains of this species could produce proficient biofilms in LB, DSM (Difco Sporulation Medium) as well and S7 medium (Figure 5). 

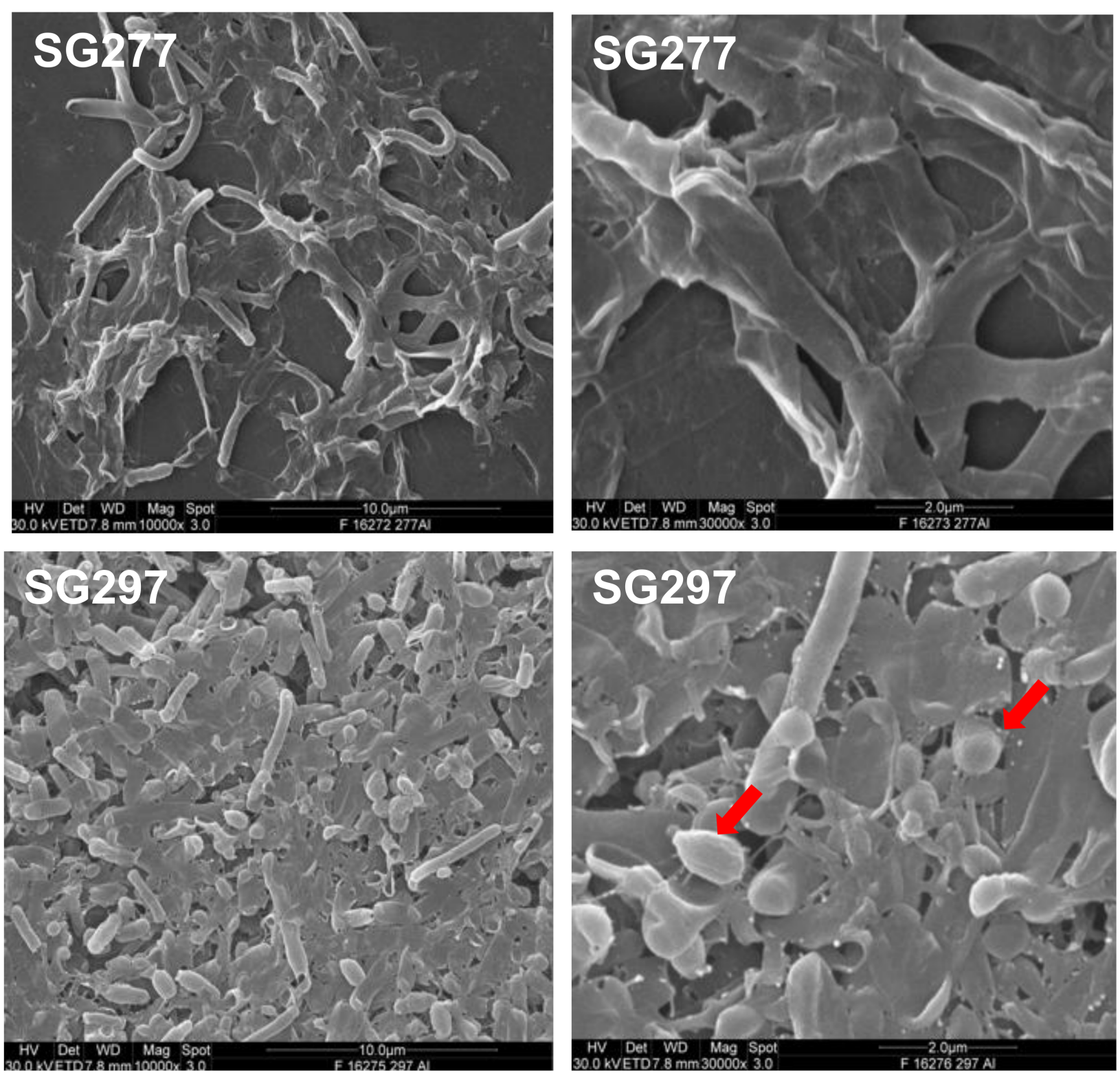

Figure 5. SG277 and SG297 biofilms. Biofilms developed in S7 medium and imaged using SEM. Red arrows highlight spores in the biofilm.

Spores were readily found in the biofilms together with vegetative cells in SG277 and other strains of B. velezensis when cultured for $18 \mathrm{~h}$ in brain heart infusion broth (BHIB). SG277 and other strains of B. velezensis demonstrated high levels of spore formation (>90\%). To test a possible effect on CD630 cells, $18 \mathrm{~h}$ cultures of SG277 grown in BHIB (containing $90 \%$ spores and vegetative cells) were pelleted, washed repeatedly and then mixed with cells of CD630 (at the logarithmic phase of growth) and plated on soft agar $(0.6 \%)$ plates. After anaerobic growth, the resulting plate exhibited complete inhibition when compared to those carrying CD630 alone (Figure 6A,B). Inhibition was also observed when a heattreated, cell-free, SG277 supernatant was used. Under these conditions of anaerobiosis, strain SG277 was unable to grow, and the most probable explanation was that the inhibitory activity was intimately associated with the cell and/or spore surface. To further investigate, PBS-washed SG277 spores $\left(\sim 5 \times 10^{8} \mathrm{CFU}\right)$ were added to a CD630 culture, rapidly lysing the cells and demonstrating that active material could also associate with the spore surface (Figure 6C). Bacteriolysis was also observed with the cell-free supernatant, the $30 \times \mathrm{AmSO}_{4}$ precipitated material, the SEC active fraction and RP-HPLC purified surfactin (Figure 6C). 
Methanol extraction of the spore surface followed by RP-HPLC analysis confirmed the presence of inhibitory lipopeptides (data not shown).

A

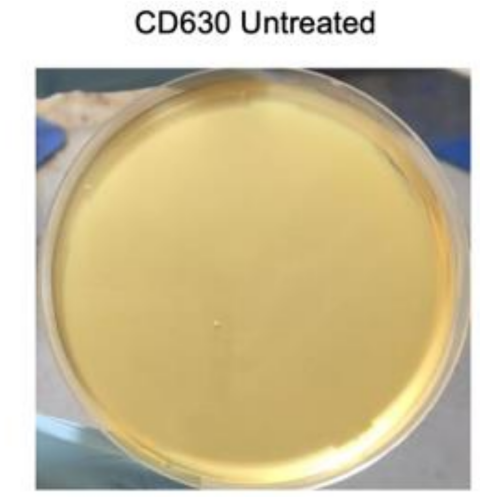

B

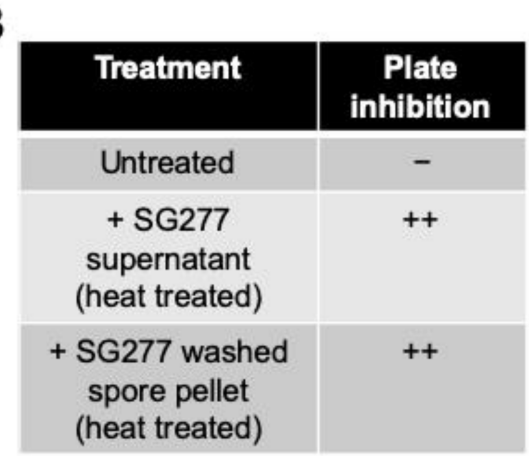

CD630 + SG277 Spores
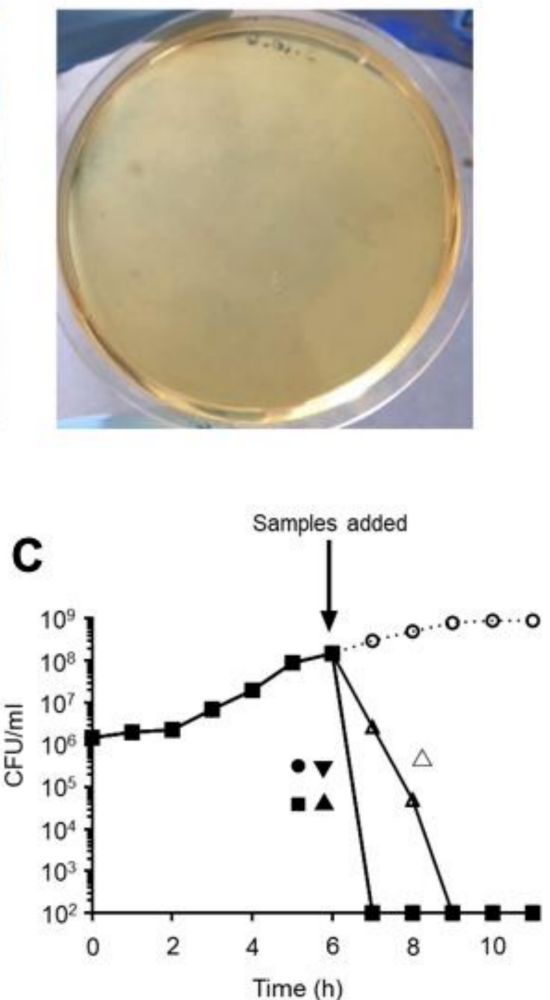

Figure 6. Inhibitory activity is associated with the surface of the cell and/or spore. (A,B) Heat-treated SG277 spores or supernatant were mixed in equal volumes with a logarithmic culture of CD630, added to $0.6 \%$ micropropagation agar and poured onto BHIS agar plates prior to anaerobic incubation $\left(37^{\circ} \mathrm{C}, 18 \mathrm{~h}\right)$. (A) Inhibition was apparent compared against the turbid appearance of untreated CD630. (B) Both the SG277 heat-treated supernatant and the spore pellet demonstrated agar plate inhibition of CD630 culture. (C) Fractions of "active" material obtained from B. velezensis SG277 were added to exponentially growing CD630 and viable bacteria (CFU/mL) were determined. Aqueous materials were: $\bullet$, cell-free supernatant; $\triangle$, spores, consisting of purified spores of SG277; $\mathbf{\square}, \mathrm{AmSO}_{4}$ precipitate; $\mathbf{\Lambda}$, SEC active fraction; $\boldsymbol{\nabla}, \mathrm{RP}-\mathrm{HPLC}$ purified surfactin; $\bigcirc, \mathrm{dH}_{2} \mathrm{O}$ (control). The activity of each fraction added was normalised prior to addition using a microdilution assay.

\subsection{Bacillus Micelles Can Incorporate Antimicrobials}

Lipopeptides are known to form micelles but the discovery of chlorotetaine in the active material from some strains (SG277 and SG297) was intriguing since the inhibitory activity was greater with this antibiotic than without. Synthetically produced micelles have been used for intracellular drug delivery $[26,27]$ and we speculated that this phenomenon could be used by Bacillus to enhance and concentrate antibacterial activity. To address this, we prepared an $\mathrm{AmSO}_{4}$ precipitate of an SG277 cell-free supernatant and mixed it with each of three antibiotics (clindamycin, vancomycin or metronidazole) that are known to inhibit the growth of $C$. difficile. Following incubation, the mixture was passed through a $10 \mathrm{kDa}$ MWCO membrane and the activity towards CD630 was determined in the eluate and retentate (Figure 7A). SG277 $\mathrm{AmSO}_{4}$ material diluted to a concentration of $\sim 0.2 \mathrm{mg} / \mathrm{mL}$ carried a basal level of CD630 inhibitory activity (1:40) that was unable to pass through the membrane due to its size ( $\sim 100 \mathrm{kDa}$.) but was retained in the retentate. For each of the three antibiotics essentially all inhibitory activity passed through the membrane as expected. For clindamycin and vancomycin, residual levels were retained possibly resulting from binding to the filtration membrane. Interestingly, the inhibitory activity to CD630 of the micelle 
fraction (i.e., the fraction unable to pass through the $10 \mathrm{kDa}$ membrane) was substantially increased when it was mixed with either clindamycin (3-fold increase) or vancomycin (2-fold increase). This increase in activity most probably results from the association of these antibiotics with the lipopeptide SG277 micelles. Lipopeptide micelles from Bacillus carry a hydrophobic interior surrounded by an anionic surface [28,29]. At physiological $\mathrm{pH}$, as used here, clindamycin and vancomycin are positively charged $[30,31]$ while metronidazole is neutral [32] possibly explaining the failure of metronidazole to interact with the micelles.

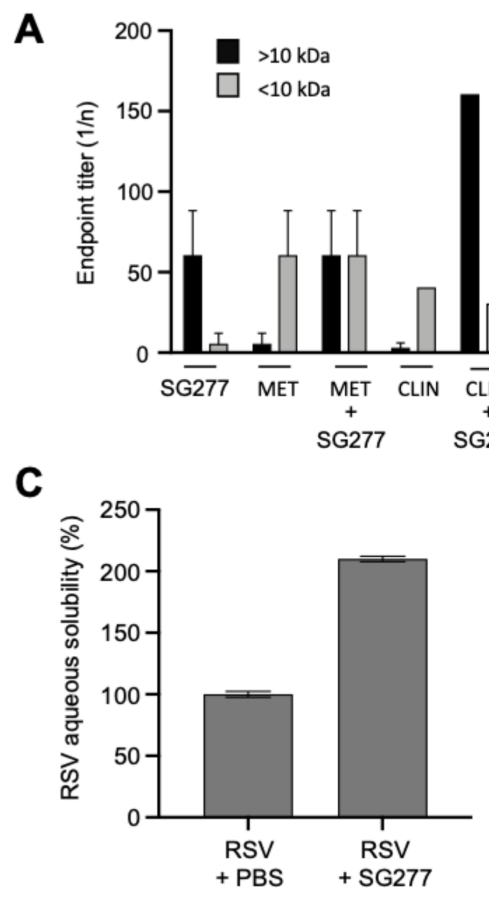

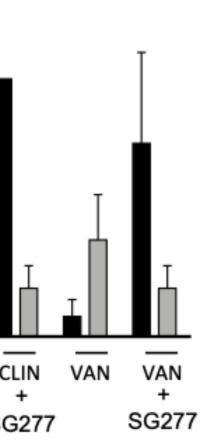

D

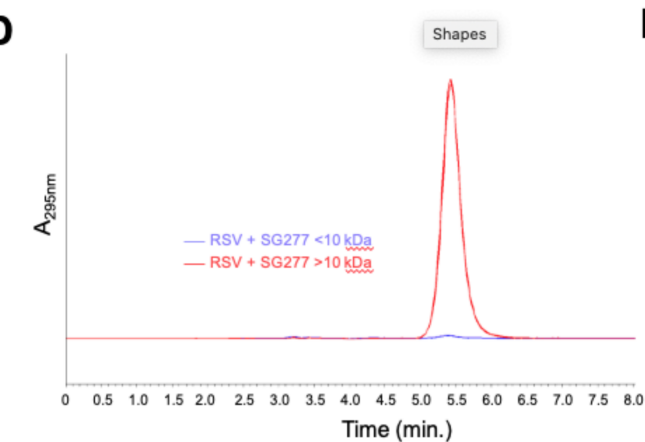

B

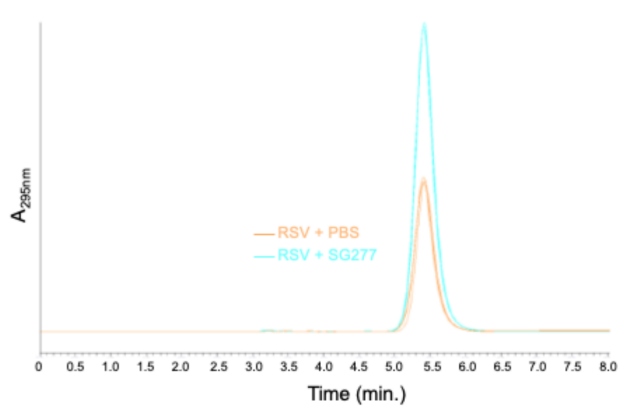

$\mathbf{E}$

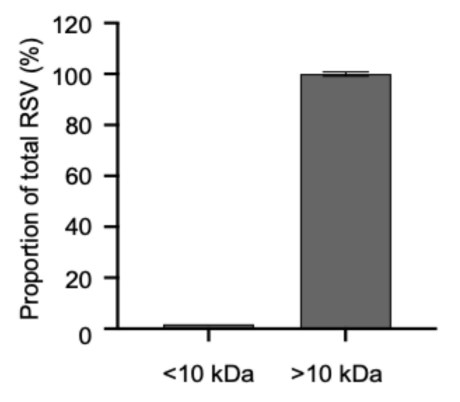

Figure 7. Micellar incorporation of antimicrobials. (A) $\mathrm{SG} 277 \mathrm{AmSO}_{4}$ material comprising micelles (mic) was mixed with either metronidazole (MET), clindamycin (CLIN) or vancomycin (VAN) and then filtered through a $10 \mathrm{kDa}$ membrane. Inhibitory activity towards CD630 in the eluate $(<10 \mathrm{kDa})$ was determined and compared to the starting solution. Antibiotics without the addition of micelles were taken through the same procedure for comparison. (B,C) SG277 SEC material or PBS were combined with resveratrol (RSV), incubated at RT and insoluble material was pelleted by centrifugation. (B) The supernatants containing solubilised resveratrol were analysed by RP-HPLC for resveratrol. (C) The absorbance was used to determine the aqueous solubility of resveratrol in SG277 SEC material as a proportion of resveratrol's solubility in PBS. The experiment was performed 3-times. (D,E) SG277 SEC material was combined with resveratrol, incubated at RT and insoluble material was pelleted by centrifugation. (D) The supernatant was then filtered through a $10 \mathrm{kDa}$ membrane and the eluate $(<10 \mathrm{kDa})$ and retentate $(>10 \mathrm{kDa})$ were analysed by RP-HPLC for resveratrol. (E) The absorbance was used to calculate the proportion of RSV in the eluate $(<10 \mathrm{kDa})$ and retentate $(>10 \mathrm{kDa})$. The experiment was performed 3-times.

Biosurfactants are frequently used to aid in the solubilisation of hydrophobic biomolecules [33], and so we asked whether SG277 micelles could perform this function. SG277 SEC material or PBS only was saturated with resveratrol, an antimicrobial polyphenolic antioxidant of clinical interest [34,35]. After incubation, the mixture was centrifuged, and the supernatant was analysed by RP-HPLC for solubilised resveratrol (Figure 7B). The presence of SG277 micelles increased the aqueous solubility of resveratrol 2-fold (Figure 7C). This increase was achieved under the experimental conditions reported, and it is probable that further study may yield optimised conditions for enhancing resveratrol solubility using SG277 micelles. Polymeric micelles have been shown to enhance the bioavailability of drugs by increasing solubility [36], and so the effect shown here illustrates the potential of Bacillus lipopeptides to increase solubility and, therefore, the bioavailability of hydrophobic antimicrobials, including resveratrol. 
Lipopeptide biosurfactants associate with other molecules via electrostatic bonding and hydrophobic interactions [37]. Hydrophobic, poorly water-soluble molecules typically interact with the interior core of micelles, as this is where the hydrophobic, lipophilic tail segments are located [38]. It follows then that in addition to increasing the solubility of resveratrol, Bacillus lipopeptides may be incorporating the hydrophobic antimicrobial into the complex. To address this, SG277 SEC material was incubated with resveratrol and following centrifugation, the supernatant containing soluble SG277 micelles + resveratrol was passed through a $10 \mathrm{kDa}$ MWCO membrane. The eluate $(<10 \mathrm{kDa})$ and the retentate $(>10 \mathrm{kDa})$ were analysed by RP-HPLC for the presence of LMW resveratrol (228 Da) (Figure 7D). Interestingly, the lipopeptide micelles retained over $99 \%$ of the LMW resveratrol and prevented it from passing through the $10 \mathrm{kDa}$ membrane demonstrating that the antimicrobial had been incorporated into the micelle (Figure 7E).

\section{Discussion}

We have shown here that $B$. velezensis is a prolific producer of antimicrobial activity. $B$. velezensis is a recently reported heterotypic synonym of B. amyloliquefaciens $[12,39]$ and shares with it several similarities, e.g., mucoid colonies and production of amylase. The description of this new species also makes it likely that many strains previously reported as $B$. amyloliquefaciens are in fact $B$. velezensis. As with other members of the $B$. subtilis group, $B$. amyloliquefaciens and $B$. velezensis produce lipopeptide antibiotics including surfactins, iturins and fengycins as well as the polyketide antibiotics, difficidin, bacillaene and macrolactins [40,41]. The lipopeptides have been well characterised in Bacillus and are known for their antibacterial [8], anti-fungal [14] and anti-viral [42,43] activity. While antimicrobial and biosurfactant activity associated with Bacillus is not unexpected, a direct link between the two is.

A key finding in this work is that lipopeptide micelles incorporate antibiotics, in this case, chlorotetaine. Numerous studies have reported on Bacillus strains with antimicrobial activity, but effort has focused on the purification of individual components rather than the whole. For example, to illustrate the efficacy of difficidin and bacilysin from B. amyloliquefaciens FZB42, analysis was made using isogenic mutants devoid of the lipopeptides surfactin, fengycin and iturins [44]. Commonly used purification procedures will break down micelles and bias effort towards the low MW species $(<2 \mathrm{kDa})$. As we have shown here, the activity of individual RP-HPLC fractions carry inhibitory activity, but this is far less than when incorporated into a mixed micelle. Accordingly, by focusing on low MW species the true activity, conferred by the micellar formulation, will most likely have been overlooked.

Micelles will form only when the concentration of individual lipopeptide monomers has exceeded the critical micelle concentration $(\mathrm{cmc}$ ) and in this form, biosurfactant properties arise [29]. In micellar form, lipopeptides efficiently disrupt not only membranes but also proteins [45]. This activity is focused on the cell and/or spore surface and probably also provides a competitive advantage in multicellular communities for enhancing motility, swarming activity and colonisation [46]. As well as entrapping and concentrating activity, the micelle may also provide a mechanism to enhance the stability of individual antimicrobials. For example, purified difficidin has been shown to have poor stability at different $\mathrm{pHs}$ and is readily oxidised [47].

Although lipopeptide micelles have been extensively studied at a biophysical level, less is known about those produced in vivo by Bacillus (and it should be emphasized that we have only examined this phenomenon in $B$. velezensis strains). We have found that the composition of lipopeptides within micelles does differ between strains and evidence is presented here that (a) a full cohort of surfactins, fengycins and iturins have greater functionality, and $(\mathrm{b})$ the length $\left(\mathrm{C}_{-\mathrm{x}}\right)$ of the hydrophobic fatty acid chain is important. Based on the results obtained, it is tempting to speculate that micelle formation may be additive, that is, micelles form from a pool of available amphiphilic molecules and the final composition of micelles will reflect the relative abundance of these lipopeptides at the 
cell surface. This might explain why in some strains we have examined (e.g., SG137 and SG185) iturins were not detected in the micelles. We also cannot rule out the inclusion of glycolipids (notably rhamnolipid) and in other work, a B. subtilis strain has been reported that carries biosurfactant activity composed of two lipopeptides (surfactin and fengycin) and glycolipids (rhamnolipids) [48].

Interaction of micellar aggregates with the hydrophilic cell surface might be expected but the spore surface is typically hydrophobic [49]. Possibly, the spores are themselves coated in bacterial exopolysaccharides derived from the culture medium and our analysis of biofilms (Figure 5) shows spores embedded in the biofilm matrix that supports this assumption. Spores have also been shown to efficiently bind lipopolysaccharides [49] and the spore may provide a suitable platform for the aggregation of lipopeptides particularly in biofilm matrices. Clearly, in multicellular communities, the accumulation of entrapped antimicrobials on the spore or cell surface may offer a competitive advantage.

Strains with the greatest activity to $C$. difficile produced micelles containing chlorotetaine. Chlorotetaine is a little-studied dipeptide antibiotic that is closely related to bacilysin [50,51]. Bacilysin is present in most members of the B. subtilis group and all strains of $B$. velezensis examined here carried a bacilysin operon yet no operon that might encode chlorotetaine was found. A single halogenation step is required to convert bacilysin to chlorotetaine [52] and it is most likely, therefore, that bacilysin is modified to chlorotetaine in strains SG277 and SG297 either using a halogenase or alternatively by exogenous chlorine.

Probably one of the primary roles of lipopeptides may be to form micelles enabling the capture of other Bacillus-produced antimicrobials. We would then expect that for strains exhibiting inhibitory activity specific to other bacteria that other micellar-antibiotic formulations might exist. It should be noted that more focused and targeted activity has also been reported for Bacillus lipopeptides. For example, with inhibitory activity to $S$. aureus. Here, B. subtilis fengycins inhibit the Agr (accessory gene regulator) quorum-sensing system by competitive inhibition with the Agr auto-inducing peptide [53]. In our work, we observed both bacteriostatic and bactericidal activity against $S$. aureus suggesting different mechanisms of action which is intriguing. We also found that for Gram-negatives only bacteriostatic activity was found while for the Gram-positive pathogens a range of activities (bacteriostatic, bactericidal and bacteriolytic) were evident. The cell envelopes of Grampositives and Gram-negatives obviously differ with the former being more susceptible to detergents [54]. Since we only examined the precise mechanism of action to $C$. difficile, we cannot exclude the possibility that for the other pathogens alternative mechanisms exist.

It seems logical to propose that micelles may have been exploited by Bacillus to improve antimicrobial performance. Recently, a comparable phenomenon has been observed in Clostridium scindens, whereby micelle-forming secondary bile acids were shown to combine with tryptophan-derived antibiotics, thereby increasing the activity against the intestinal pathogen C. difficile [55]. It should be emphasised that individual lipopeptides, as well as chlorotetaine, do show activity to $C$. difficile, but this is less than when combined (Figure 3). One apparent anomaly then is our observation that when fractionated by SDS-PAGE an active species of $1 \mathrm{kDa}$ showed in situ activity to CD630 (Figure 2B). We cannot correlate the level of this activity to those determined through a microdilution assay, but one possibility is that the lipopeptides re-form into high molecular weight micelles following the removal of SDS, which is able to disrupt intermolecular bonding [56].

One surprising observation made here is the presence of large aggregates $20-50 \mathrm{~nm}$ in size. These granular clusters were primarily larger than the $10 \mathrm{~nm}$ micelles observed by cryo-EM and potentially represent supermicelles formed by supramolecular assembly [57]. DLS analysis showed evidence of a large aggregate size and possibly these aggregates might be easily entrapped in the exopolysaccharide layers of the cell envelope.

In summary, we have demonstrated the superior antimicrobial activity of a naturally occurring mixture of lipopeptides, as compared with a single lipopeptide species. Solubilisation of drugs using synthetic micelles [58] is a promising therapeutic approach now 
being developed and so it is useful to consider whether Bacillus lipopeptides could be exploited for nanoparticle micelle drug delivery.

Supplementary Materials: The following are available online at https:/ / www.mdpi.com/article/ 10.3390 / pharmaceutics13081296/s1, Figure S1: Pathogen growth kinetics using CFU counts, Figure S2: MALDI-TOF analysis of commercial vs Bacillus surfactins, Figure S3: Purification steps, Table S1: Genome analysis of lipopeptide, polyketide and bacilysin genes present in B. velezensis "active" strains., Table S2: Antimicrobial screening.

Author Contributions: Conceptualization, S.M.C., H.A.H., M.S. and W.T.F.; methodology, S.M.C., M.S., H.A.H. and W.T.F.; software, W.T.F. and M.H.; validation, W.T.F., H.A.H., M.H., J.R.G.A. and M.S.; formal analysis, W.T.F., H.A.H., M.H., J.R.G.A., H.W., K.B., S.T., L.B., E.F., A.B., M.T.R., W.J.J.M. and M.S.; investigation, W.T.F., H.A.H., M.H., J.R.G.A., H.W., K.B., S.T., L.B., E.F., A.B., E.R., M.T.R., W.J.J.M. and M.S.; resources, S.M.C., L.B., E.F., A.B., E.R., W.J.J.M. and M.S.; data curation, W.T.F., M.H., J.R.G.A., H.W., K.B., S.T., L.B., E.F., A.B., E.R., M.T.R., W.J.J.M. and M.S.; writing-original draft preparation, S.M.C., W.T.F.; writing-review and editing, S.M.C., W.T.F., M.H., J.R.G.A., E.R., W.J.J.M. and M.S.; visualization, W.T.F., S.M.C., M.H. and M.S.; supervision, S.M.C. and M.S.; project administration, S.M.C.; funding acquisition, S.M.C. and W.J.J.M. All authors have read and agreed to the published version of the manuscript.

Funding: This work was supported by grants from the Medical Research Council (MR/R026262/1) to SMC and the Ministry of Economy and Competitiveness of the Spanish Government PID2019_108778GB_C21 (AEI/FEDER, EU) to W.J.J.M.

Institutional Review Board Statement: Not applicable.

Informed Consent Statement: Not applicable.

Data Availability Statement: The data presented in this study are available from the corresponding author upon reasonable request.

Conflicts of Interest: S.M.C. and H.A.H. are shareholders in SporeGen Ltd. S.M.C. is a director at SporeGen Ltd. H.W. and K.B. are employees (research technicians) at SporeGen Ltd. The funders had no role in the design of the study; in the collection, analyses, or interpretation of data; in the writing of the manuscript, or in the decision to publish the results. The company played a role in the collection, analyses, or interpretation of data.

\section{References}

1. Nicholson, W.L. Roles of Bacillus endospores in the environment. Cell. Mol. Life Sci. 2002, 59, 410-416. [CrossRef]

2. Burrows, S.M.; Elbert, W.; Lawrence, M.G.; Poschl, U. Bacteria in the global atmosphere-Part 1: Review and synthesis of literature data for different ecosystems. Atmos. Chem. Phys. 2009, 9, 9263-9280. [CrossRef]

3. Tam, N.K.; Uyen, N.Q.; Hong, H.A.; Duc, L.H.; Hoa, T.T.; Serra, C.R.; Henriques, A.O.; Cutting, S.M. The intestinal life cycle of Bacillus subtilis and close relatives. J. Bacteriol. 2006, 188, 2692-2700. [CrossRef] [PubMed]

4. Hong, H.A.; Khaneja, R.; Tam, N.M.; Cazzato, A.; Tan, S.; Urdaci, M.; Brisson, A.; Gasbarrini, A.; Barnes, I.; Cutting, S.M. Bacillus subtilis isolated from the human gastrointestinal tract. Res. Microbiol. 2009, 160, 134-143. [CrossRef]

5. Caulier, S.; Nannan, C.; Gillis, A.; Licciardi, F.; Bragard, C.; Mahillon, J. Overview of the Antimicrobial Compounds Produced by Members of the Bacillus subtilis Group. Front. Microbiol. 2019, 10, 302. [CrossRef]

6. Stein, T. Bacillus subtilis antibiotics: Structures, syntheses and specific functions. Mol. Microbiol. 2005, 56, 845-857. [CrossRef] [PubMed]

7. Abriouel, H.; Franz, C.M.; Ben Omar, N.; Galvez, A. Diversity and applications of Bacillus bacteriocins. FEMS Microbiol. Rev. 2011, 35, 201-232. [CrossRef] [PubMed]

8. Sumi, C.D.; Yang, B.W.; Yeo, I.C.; Hahm, Y.T. Antimicrobial peptides of the genus Bacillus: A new era for antibiotics. Can. J. Microbiol. 2015, 61, 93-103. [CrossRef]

9. Mnif, I.; Ghribi, D. Review lipopeptides biosurfactants: Mean classes and new insights for industrial, biomedical, and environmental applications. Biopolymers 2015, 104, 129-147. [CrossRef]

10. Nayarisseri, A.; Singh, P.; Singh, S.K. Screening, isolation and characterization of biosurfactant producing Bacillus subtilis strain ANSKLAB03. Bioinformation 2018, 14, 304-314. [CrossRef]

11. Hsueh, Y.H.; Somers, E.B.; Wong, A.C. Characterization of the codY gene and its influence on biofilm formation in Bacillus cereus. Arch. Microbiol. 2008, 189, 557-568. [CrossRef] [PubMed]

12. Fan, B.; Blom, J.; Klenk, H.P.; Borriss, R. Bacillus amyloliquefaciens, Bacillus velezensis, and Bacillus siamensis Form an “Operational Group B. amyloliquefaciens" within the B. subtilis Species Complex. Front. Microbiol. 2017, 8, 22. [CrossRef] 
13. Meena, K.R.; Kanwar, S.S. Lipopeptides as the antifungal and antibacterial agents: Applications in food safety and therapeutics. BioMed Res. Int. 2015, 2015, 473050. [CrossRef]

14. Ongena, M.; Jacques, P. Bacillus lipopeptides: Versatile weapons for plant disease biocontrol. Trends Microbiol. 2008, 16, 115-125. [CrossRef] [PubMed]

15. Phister, T.G.; O'Sullivan, D.J.; McKay, L.L. Identification of bacilysin, chlorotetaine, and iturin a produced by Bacillus sp. strain CS93 isolated from pozol, a Mexican fermented maize dough. Appl. Environ. Microbiol. 2004, 70, 631-634. [CrossRef]

16. Karatan, E.; Michael, A.J. A wider role for polyamines in biofilm formation. Biotechnol. Lett. 2013, 35, 1715-1717. [CrossRef]

17. Deleu, M.; Bouffioux, O.; Razafindralambo, H.; Paquot, M.; Hbid, C.; Thonart, P.; Jacques, P.; Brasseur, R. Interaction of surfactin with membranes: A computational approach. Langmuir 2003, 19, 3357-3385. [CrossRef]

18. Kracht, M.; Rokos, H.; Ozel, M.; Kowall, M.; Pauli, G.; Vater, J. Antiviral and hemolytic activities of surfactin isoforms and their methyl ester derivatives. J. Antibiot. 1999, 52, 613-619. [CrossRef]

19. Bechet, M.; Caradec, T.; Hussein, W.; Abderrahmani, A.; Chollet, M.; Leclere, V.; Dubois, T.; Lereclus, D.; Pupin, M.; Jacques, P. Structure, biosynthesis, and properties of kurstakins, nonribosomal lipopeptides from Bacillus spp. Appl. Microbiol. Biotechnol. 2012, 95, 593-600. [CrossRef] [PubMed]

20. Ma, Z.; Wang, N.; Hu, J.; Wang, S. Isolation and characterization of a new iturinic lipopeptide, mojavensin A produced by a marine-derived bacterium Bacillus mojavensis B0621A. J. Antibiot. 2012, 65, 317-322. [CrossRef] [PubMed]

21. Chen, X.H.; Vater, J.; Piel, J.; Franke, P.; Scholz, R.; Schneider, K.; Koumoutsi, A.; Hitzeroth, G.; Grammel, N.; Strittmatter, A.W.; et al. Structural and functional characterization of three polyketide synthase gene clusters in Bacillus amyloliquefaciens FZB 42. J. Bacteriol. 2006, 188, 4024-4036. [CrossRef] [PubMed]

22. Goodson, J.R.; Klupt, S.; Zhang, C.; Straight, P.; Winkler, W.C. LoaP is a broadly conserved antiterminator protein that regulates antibiotic gene clusters in Bacillus amyloliquefaciens. Nat. Microbiol. 2017, 2, 17003. [CrossRef] [PubMed]

23. Zimmerman, S.B.; Schwartz, C.D.; Monaghan, R.L.; Pelak, B.A.; Weissberger, B.; Gilfillan, E.C.; Mochales, S.; Hernandez, S.; Currie, S.A.; Tejera, E.; et al. Difficidin and oxydifficidin: Novel broad spectrum antibacterial antibiotics produced by Bacillus subtilis. I. Production, taxonomy and antibacterial activity. J. Antibiot. 1987, 40, 1677-1681. [CrossRef]

24. Ozcengiz, G.; Alaeddinoglu, N.G.; Demain, A.L. Regulation of biosynthesis of bacilysin by Bacillus subtilis. J. Ind. Microbiol. 1990, 6, 91-100. [CrossRef] [PubMed]

25. Steinborn, G.; Hajirezaei, M.R.; Hofemeister, J. bac genes for recombinant bacilysin and anticapsin production in Bacillus host strains. Arch. Microbiol. 2005, 183, 71-79. [CrossRef]

26. Chen, M.; Xie, S.; Wei, J.; Song, X.; Ding, Z.; Li, X. Antibacterial Micelles with Vancomycin-Mediated Targeting and pH/LipaseTriggered Release of Antibiotics. ACS Appl. Mater. Interfaces 2018, 10, 36814-36823. [CrossRef]

27. Hong, W.; Chen, D.; Zhang, X.; Zeng, J.; Hu, H.; Zhao, X.; Qiao, M. Reversing multidrug resistance by intracellular delivery of Pluronic(R) P85 unimers. Biomaterials 2013, 34, 9602-9614. [CrossRef]

28. Jauregi, P.; Coutte, F.; Catiau, L.; Lecouturier, D.; Jacques, P. Micelle size characterization of lipopeptides produced by B. subtilis and their recovery by the two-step ultrafiltration process. Sep. Purif. Technol. 2013, 104, 175-182. [CrossRef]

29. HamLey, I.W.; Dehsorkhi, A.; Jauregi, P.; Seitsonen, J.; Ruokolainen, J.; Coutte, F.; Chataigne, G.; Jacques, P. Self-assembly of three bacterially-derived bioactive lipopeptides. Soft Matter 2013, 9, 9572-9578. [CrossRef]

30. Johnson, J.L.; Yalkowsky, S.H. Reformulation of a new vancomycin analog: An example of the importance of buffer species and strength. AAPS PharmSciTech 2006, 7, E33-E37. [CrossRef]

31. Wishart, D.S.; Feunang, Y.D.; Marcu, A.; Guo, A.C.; Liang, K.; Vazquez-Fresno, R.; Sajed, T.; Johnson, D.; Li, C.; Karu, N.; et al. HMDB 4.0: The human metabolome database for 2018. Nucleic Acids Res. 2018, 46, D608-D617. [CrossRef] [PubMed]

32. Wishart, D.S.; Knox, C.; Guo, A.C.; Shrivastava, S.; Hassanali, M.; Stothard, P.; Chang, Z.; Woolsey, J. DrugBank: A comprehensive resource for in silico drug discovery and exploration. Nucleic Acids Res. 2006, 34, D668-D672. [CrossRef]

33. Thakur, S.; Singh, A.; Sharma, R.; Aurora, R.; Jain, S.K. Biosurfactants as a Novel Additive in Pharmaceutical Formulations: Current Trends and Future Implications. Curr. Drug Metab. 2020, 21, 885-901. [CrossRef] [PubMed]

34. Vestergaard, M.; Ingmer, H. Antibacterial and antifungal properties of resveratrol. Int. J. Antimicrob. Agents 2019, 53, 716-723. [CrossRef] [PubMed]

35. Berman, A.Y.; Motechin, R.A.; Wiesenfeld, M.Y.; Holz, M.K. The therapeutic potential of resveratrol: A review of clinical trials. NPJ Precis. Oncol. 2017, 1, 35. [CrossRef] [PubMed]

36. Xu, W.; Ling, P.; Zhang, T. Polymeric micelles, a promising drug delivery system to enhance bioavailability of poorly water-soluble drugs. J. Drug Deliv. 2013, 2013, 340315. [CrossRef]

37. Bai, G.; Nichifor, M.; Lopes, A.; Bastos, M. Thermodynamics of self-assembling of hydrophobically modified cationic polysaccharides and their mixtures with oppositely charged surfactants in aqueous solution. J. Phys. Chem. B 2005, 109, 21681-21689. [CrossRef]

38. Croy, S.R.; Kwon, G.S. Polymeric micelles for drug delivery. Curr. Pharm. Des. 2006, 12, 4669-4684. [CrossRef]

39. Wang, L.T.; Lee, F.L.; Tai, C.J.; Kuo, H.P. Bacillus velezensis is a later heterotypic synonym of Bacillus amyloliquefaciens. Int. J. Syst. Evol. Microbiol. 2008, 58 Pt 3, 671-675. [CrossRef]

40. Harwood, C.R.; Mouillon, J.M.; Pohl, S.; Arnau, J. Secondary metabolite production and the safety of industrially important members of the Bacillus subtilis group. FEMS Microbiol. Rev. 2018, 42, 721-738. [CrossRef] 
41. Arguelles-Arias, A.; Ongena, M.; Halimi, B.; Lara, Y.; Brans, A.; Joris, B.; Fickers, P. Bacillus amyloliquefaciens GA1 as a source of potent antibiotics and other secondary metabolites for biocontrol of plant pathogens. Microb. Cell Factories 2009, 8, 63. [CrossRef]

42. Vollenbroich, D.; Ozel, M.; Vater, J.; Kamp, R.M.; Pauli, G. Mechanism of inactivation of enveloped viruses by the biosurfactant surfactin from Bacillus subtilis. Biol. J. Int. Assoc. Biol. Stand. 1997, 25, $289-297$.

43. Jung, M.; Lee, S.; Kim, H. Recent studies on natural products as anti-HIV agents. Curr. Med. Chem. 2000, 7, 649-661. [CrossRef] [PubMed]

44. Wu, L.; Wu, H.; Chen, L.; Yu, X.; Borriss, R.; Gao, X. Difficidin and bacilysin from Bacillus amyloliquefaciens FZB42 have antibacterial activity against Xanthomonas oryzae rice pathogens. Sci. Rep. 2015, 5, 12975. [CrossRef]

45. Otzen, D.E. Biosurfactants and surfactants interacting with membranes and proteins: Same but different? Biochim. Biophys. Acta Biomembr. 2017, 1859, 639-649. [CrossRef] [PubMed]

46. Ghelardi, E.; Salvetti, S.; Ceragioli, M.; Gueye, S.A.; Celandroni, F.; Senesi, S. Contribution of surfactin and SwrA to flagellin expression, swimming, and surface motility in Bacillus subtilis. Appl. Environ. Microbiol. 2012, 78, 6540-6544. [CrossRef] [PubMed]

47. Farrell, L.J.; Lo, R.; Wanford, J.J.; Jenkins, A.; Maxwell, A.; Piddock, L.J.V. Revitalizing the drug pipeline: AntibioticDB, an open access database to aid antibacterial research and development. J. Antimicrob. Chemother. 2018, 73, 2284-2297. [CrossRef]

48. Cheng, F.; Tang, C.; Yang, H.; Yu, H.; Chen, Y.; Shen, Z. Characterization of a blend-biosurfactant of glycolipid and lipopeptide produced by Bacillus subtilis TU2 isolated from underground oil-extraction wastewater. J. Microbiol. Biotechnol. 2013, 23, 390-396. [CrossRef]

49. Huang, J.M.; Hong, H.A.; Van Tong, H.; Hoang, T.H.; Brisson, A.; Cutting, S.M. Mucosal delivery of antigens using adsorption to bacterial spores. Vaccine 2010, 28, 1021-1030. [CrossRef]

50. Kenig, M.; Abraham, E.P. Antimicrobial activities and antagonists of bacilysin and anticapsin. J. Gen. Microbiol. 1976, 94, 37-45. [CrossRef]

51. Rapp, C.; Jung, G.; Katzer, W.; Loeffler, W. Chlorotetain from Bacillus subtilis, an antifungal dipeptide with an unusual chlorinecontaining amino acid. Angew. Chem. Int. Ed. Engl. 1988, 27, 1733-1734. [CrossRef]

52. Wild, H. Enantioselective total synthesis of the antifungal natural products Chlorotetaine, Bacilysisn, and Anticapsin and of related compounds: Revision of the relative configuration. J. Org. Chem 1994, 59, 2748-2761. [CrossRef]

53. Piewngam, P.; Zheng, Y.; Nguyen, T.H.; Dickey, S.W.; Joo, H.S.; Villaruz, A.E.; Glose, K.A.; Fisher, E.L.; Hunt, R.L.; Li, B.; et al. Pathogen elimination by probiotic Bacillus via signalling interference. Nature 2018, 562, 532-537. [CrossRef] [PubMed]

54. Baker, Z.; Harrison, R.W.; Miller, B.F.; Technical Assistance of Robert Wexler. The Bactericidal Action of Synthetic Detergents. J. Exp. Med. 1941, 74, 611-620. [CrossRef]

55. Kang, J.D.; Myers, C.J.; Harris, S.C.; Kakiyama, G.; Lee, I.K.; Yun, B.S.; Matsuzaki, K.; Furukawa, M.; Min, H.K.; Bajaj, J.S.; et al. Bile Acid 7alpha-Dehydroxylating Gut Bacteria Secrete Antibiotics that Inhibit Clostridium difficile: Role of Secondary Bile Acids. Cell Chem. Biol. 2019, 26, 27-34.e4. [CrossRef] [PubMed]

56. Liu, K.; Yang, L.; Peng, X.; Wang, J.; Lu, J.R.; Xu, H. Modulation of Antimicrobial Peptide Conformation and Aggregation by Terminal Lipidation and Surfactants. Langmuir 2020, 36, 1737-1744. [CrossRef] [PubMed]

57. Li, X.; Gao, Y.; Boott, C.E.; Winnik, M.A.; Manners, I. Non-covalent synthesis of supermicelles with complex architectures using spatially confined hydrogen-bonding interactions. Nat. Commun. 2015, 6, 8127. [CrossRef]

58. Rangel-Yagui, C.O.; Pessoa, A., Jr.; Tavares, L.C. Micellar solubilization of drugs. J. Pharm. Pharm. Sci. 2005, 8, 147-165. 